Published in:

Gamito, S. 2010. Caution is needed when applying Margalef diversity index. Ecological Indicators 10: 550-551.

\title{
Caution is needed when applying Margalef Diversity Index
}

Gamito, Sofia

IMAR - Faculty of Sciences and Technology, Universidade do Algarve, Campus de Gambelas, 8005139 Faro, Portugal

\section{Abstract}

The use of diversity indices has increased due to the necessity of testing different methodologies to develop the ecological status classification of water bodies within the water framework directive implementation. The Margalef diversity index is one of the indices applied within these aims. Several software packages calculate various diversity indices. However, these packages do not give any warning that for the Margalef index the data must be organized as absolute numbers and not as a density data matrix. In data expressed as number of individuals per square meter, if the sample size is lower than a square meter this index is sub estimated. With other diversity indices commonly used in ecological evaluation it is indifferent to use density data or absolute numbers since these indices only consider relative proportions in their calculus.

Keywords: Margalef diversity index, Sampling area, Water framework directive.

\section{Introduction}

An increasing number of papers have been published in scientific journals testing the use of different indices and combination of indices after the Water framework directive publication (see for example Gamito, 2008; Pinto et al., 2009, Salas et al., 2006).

The Margalef diversity index (Margalef, 1958) (d) can easily be calculated in a spreadsheet: $d=(S-1) / \ln N$ 
Where $S$ is the number of species, and $N$ is the total number of individuals in the sample. There are, however, popular software packages, such as PRIMER and PAST, which allow the simultaneous calculation of several diversity indices. Some of this software calculates the Margalef diversity if a density data set is introduced instead of absolute numbers.

A data set that resulted from the compilation of several projects (Gamito and Furtado, 2009) was used to test the behaviour of Margalef index if absolute numbers are consider or, instead, densities.

\section{Methods}

A data set of subtidal macroinvertebrate samples from Ria Formosa coastal lagoon (Gamito and Furtado, 2009) was used. Different teams, although using comparable sampling methodology and laboratory procedures, sampled areas of different sizes, from 0.05 to $0.3 \mathrm{~m}^{-2}$.

The data was organized in two matrices, one of absolute numbers (total number of individuals of each species in each sampling station / sampling occasion) and the second as densities (number of individuals per square meter in each sampling station / sampling occasion).

The species richness (total number of species in each sample), and Margalef index, considering either the absolute number of individuals or the density, were calculated. The percentage variation was calculated as the ratio of Margalef index determined with the density matrix divided by the Margalef index determined with the absolute numbers matrix.

\section{Results}

When the density matrix was used the Margalef index was always lower then when the absolute numbers were used (Figs. 1 and 2). In fact, the index is always sub estimated with the density matrix and the difference is larger with smaller sample sizes (Fig. 3). There is an almost perfect linear relationship between the Margalef index and species richness (Fig. 2) with the exception of species richness between 20 and 40. This interval corresponds to the values observed in stations 6 to 10. In these stations, although the number of species was not very high (Figure 1), the number of individuals was low when compared with other stations. 


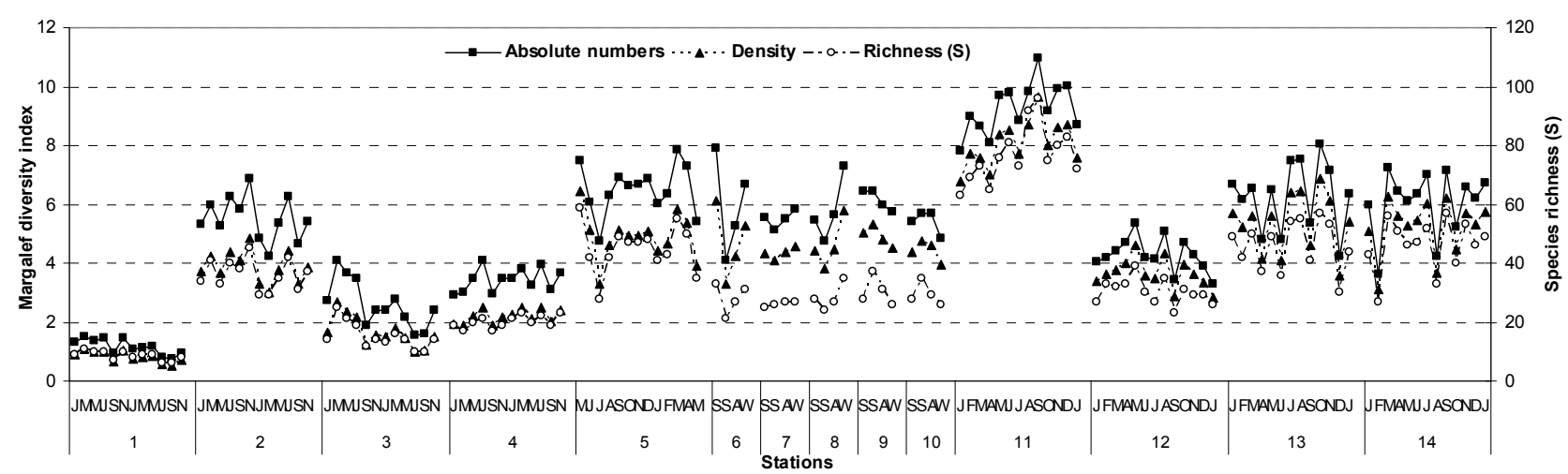

Fig. 1. Variation of macroinvertebrate species richness in 14 subtidal stations of Ria Formosa, sampled at several occasions (letters represent the sampled months). Variation of the Margalef diversity index in the same stations, considering absolute numbers or densities.

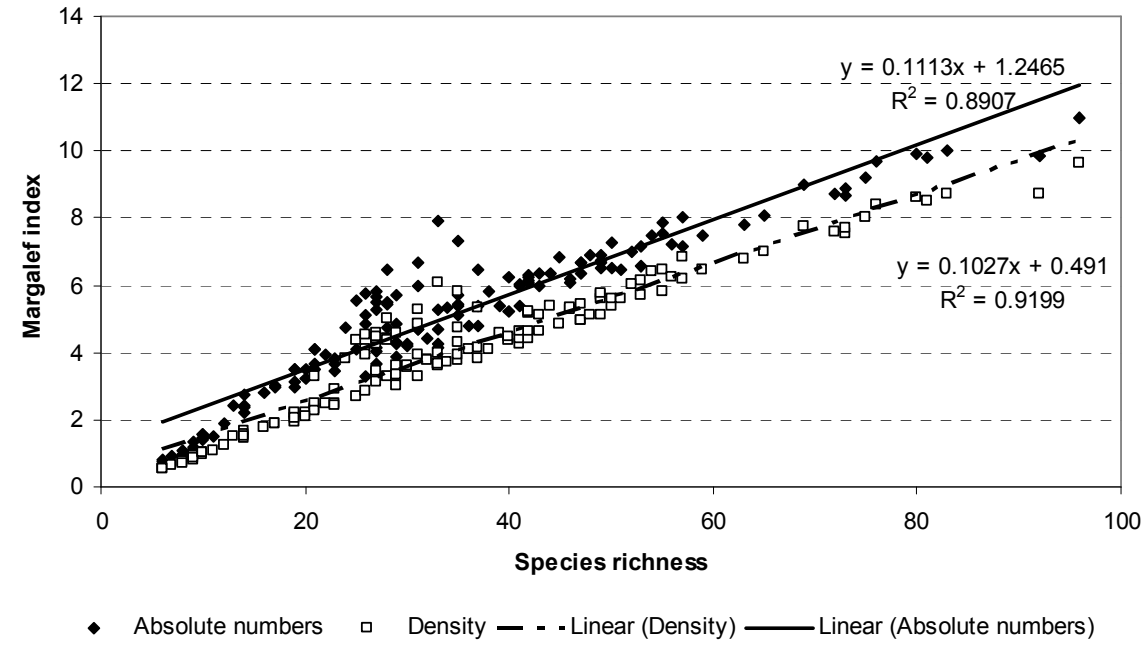

Fig. 2. Relation between Margalef index and species richness.

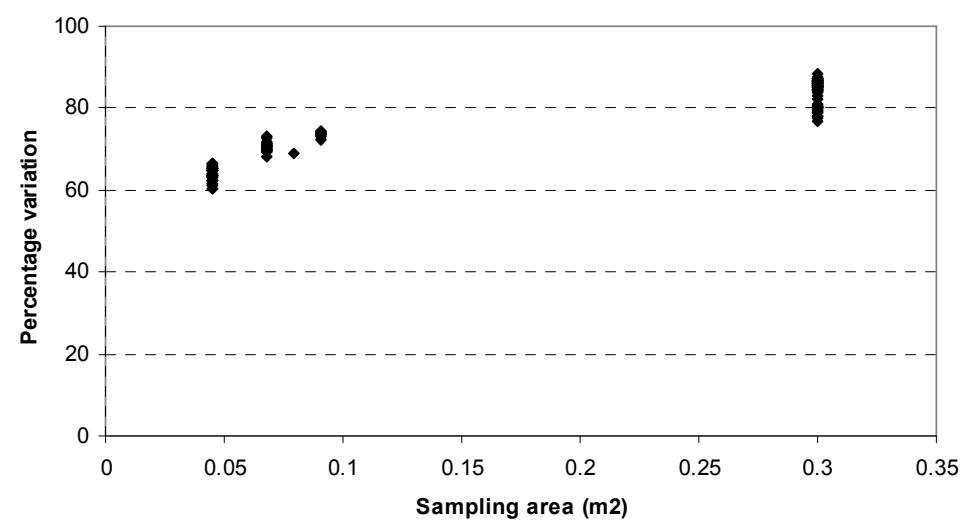

Fig. 3. Percentage variation with sampling area of Margalef index if calculated with a density matrix instead of with a matrix of absolute numbers. 


\section{Discussion}

The Margalef index measures species richness and it is highly sensitive to sample size although it tries to compensate for sampling effects (Magurran, 2004). It is a very simple index to apply that can be use in conjunction with indices sensitive to evenness or changes in dominant species, such as the dominance Berger-Parker index (Berger and Parker, 1970).

Nevertheless, software users need to be cautious when using Margalef index, as the results are very different if densities are used instead of total numbers. For other commonly used diversity indices such as Shannon-Wiener (Shannon and Weaver, 1963) and Simpson (Simpson, 1949), as these indices consider proportions and not absolute numbers, there is no difference in using either of the data sets. For Margalef index there is a sub estimation of the index.

\section{Acknowledgements}

The author thanks Ramila Furtado for helping with data matrix handling. The present study was partially carried out in the scope of EFICAS (POCI/MAR/61324/2004).

\section{References}

Berger, W.H., Parker, F.L. 1970. Diversity of planktonic foraminifera in deep sea sediments. Science 168, 1345-1347.

Gamito, S. 2008. Water Framework Directive: Defining the Ecological Quality Status in Transitional and Coastal Waters. In I.E. Gonenç, A. Vadineanu, J.P., Wolflin, R.C. Russo, Sustainable use and development of watersheds. Springer Science, pp. 323-335.

Gamito, S. and Furtado, R. 2009. Feeding diversity in macroinvertebrate communities: A contribution to estimate the ecological status in shallow waters. Ecological Indicators, 9: 1009-1019.

Magurran, A.E. 2004. Measuring biological diversity. Blackwell Publishing, London.

Margalef, R. 1958. Information theory in ecology. Gen. Systems 3, 36-71.

Pinto, R., Patrício, J., Baeta, A., Fath, B., Neto, J.M., Marques, J.C. 2009. Review and evaluation of estuarine biotic indices to assess benthic condition. Ecological Indicators 9, 1-25.

Salas, F., Marcos, C., Neto, J.M., Patrício, J., Pérez-Ruzafa, A., Marques, J.C. 2006. User-friendly guide for using benthic ecological indicators in coastal and marine quality assessment. Ocean \& Coastal Management 49, 308-331.

Shannon, C.E., Weaver, W. 1963. The Mathematical Theory of Communication. The University of Illinois Press, Illinois.

Simpson, E.H. 1949. Measurement of diversity. Nature 163, 688. 